\title{
用于深度学习训练加速的自适应框架设计
}

\author{
范涛 ${ }^{1)}$, 㚞平 ${ }^{2)}$, 成元庆 ${ }^{1 *}$ \\ 1) (北京航空航天大学集成电路科学与工程学院 北京 100191) \\ 2) (北京深维科技有限公司 北京 100191) \\ (yuanqing@ieee.org)
}

\begin{abstract}
摘 要: 用 FPGA 加速深度学习算法的训练过程通常需要较长的开发周期和丰富的硬件设计经验. 为了应对这一挑 战，设计了一种基于自适应模板技术的深度学习算法训练加速框架，在应用规模、并行调度策略、资源使用和功能扩 展上进行了深人的研究并提出了相应的优化策略. 采用 CPU-FPGA 异构加速模板技术，提出了自适应的上层模型编 译框架实现与不同硬件加速资源的适配. 这种基于定制模板的软硬件协同设计可以很好地适配不同的 FPGA 芯片并 支持算法的快速迭代. 用图神经网络算法数据进行加速对比实验, 实现了与 CPU 相比 7 41 倍的速度提升.
\end{abstract}

关键词: 深度学习; 图神经网络; 异构加速器; 现场可编程门阵列

中图法分类号: TP391.41ＤOI: 10.3724/SP.J.1089.2021.18587

\section{Template-Based Adaptive Training Acceleration Framework for Deep Learning Algorithms}

\author{
Fan Tao ${ }^{1)}$, Fan Ping ${ }^{2)}$, and Cheng Yuanqing ${ }^{1)^{*}}$ \\ 1) (School of Integrated Circuit Science and Engineering, Beihang University, Beijing 100191) \\ 2) (DeePoly Technology Inc., Beijing 100191)
}

\begin{abstract}
Field-programmable gate array (FPGA) is usually used to accelerate the training phase of deep learning algorithms, but it usually requires a long development cycle and rich hardware design expertise for satisfied execution performance. In order to deal with this challenge, an adaptive acceleration framework for deep learning algorithm is proposed in this paper. We investigate the application scale, parallel scheduling strategy, resource usage and the scalability of functionality. With the CPU-FPGA heterogeneous acceleration template based technology, an adaptive model compiler is proposed to customize the accelerator based on the algorithm's complexity and hardware resources available. The proposed hardware and software co-design framework can effectively adapt to different FPGA hardware resources and support the fast evolution of deep learning algorithms. Taking the graph neural network as an example, it can obtain 7 41x performance improvements compared to the general purpose CPU platform.
\end{abstract}

Key words: deep learning; graph convolutional networks (GCN); heterogeneous accelerator; field-programmable gate array (FPGA)

收稿日期：2020-08-15; 修回日期：2020-12-22. 基金项目：北京市自然科学基金(4192035); 北京市首都科技创新项目 (XM20200028-C). 范涛(1998一), 男, 硕士研究生, 主要研究方向为数字集成电路设计; 㚞平(1979一), 男, 高级工程师, 主要研究方 向为神经网络加速器设计和优化; 成元庆(1981一), 男, 博士, 助理教授, 硕士生导师, CCF 高级会员, 论文通讯作者, 主要研究方向 为三维集成电路设计自动化、低功耗和高可靠计算机体系结构设计. 
近年来，随着新型神经网络的出现，深度学习 算法的结构越来越复杂. 这些复杂模型包含多达数 十亿个操作和数百万个参数, 训练和部署这些模型 需要大量的计算资源 ${ }^{[1-4]}$. 这对通用处理器 (general purpose processor, GPP)是一个巨大挑战. 一些新兴 的基于图形处理器(graphics processing unit, GPU)、 现场可编程逻辑门阵列 (field programmable gate array，FPGA)和专用集成电路(application specific integrated circuit, ASIC)的硬件加速技术不断涌现 ${ }^{[5]}$.

通常，在评估硬件平台的加速效果时，必须考 虑灵活性和性能之间的权衡.一方面, GPP 可提供 高度的灵活性和易用性，但效率低下; 另一方面， ASIC 可提供高性能, 但不够灵活且开发周期长、 开发难度大 ${ }^{[6-8]}$

FPGA 是这 2 个极端之间的折中 ${ }^{[9]}$ ，它属于一 类更通用的可编程逻辑设备. FPGA 的可编程逻辑 元件可用于实现普通逻辑功能中的数据和控制通 路, 而不依赖于冯. 诺伊曼架构. 它们也能够利用 分布式片上存储器，以及深度流水线实现并行，这 与深度学习方法自然契合. 再者, 在进行机器学习 和深度学习算法开发时, 较少强调算法与硬件结 构的绑定，从而留出更多的自由度去探索算法层 面的优化. 然而，上层算法快速迭代所需的下层复 杂逻辑硬件实现对 ASIC 芯片设计提出了巨大挑 战，但 FPGA 可以很灵活地满足算法迭代的需求. 因此, FPGA 既能提供 ASIC 的性能优势, 又具备 GPP 的灵活性 ${ }^{[10-14]}$.

目前, 有很多将 FPGA 应用于机器学习加速的 工作 ${ }^{[15-20]}$, 且加速效果显著. 功能不断强化的 FPGA 设计工具使其对机器学习中经常使用的上 层算法兼容性更强，从而使 FPGA 更容易为模型搭
建和部署者所采用. 而且 FPGA 架构灵活, 使研究 者能够在诸如 GPU 的固定架构之外进行模型优化. 但是 FPGA 的开发周期较长, 需要具有丰富经验的 FPGA 硬件工程师. 伴随各种机器学习算法的不断 提出, 其中一些算法的存在周期也很短暂, 如何提 出一种可推广的、研发周期短的加速器框架是当下 的一个挑战.

\section{TABLA 平台简介}

TABLA 机器学习加速框架 ${ }^{[15]}$ 实现了利用自适 应模板技术对机器学习训练过程的加速.

如图 1 所示, TABLA 旨在从编程模式上提供 一个全面的解决方案, 能够自动地生成针对一类 机器学习算法的训练阶段的加速器架构.

(1) 高级编程模型. TABLA 提供了一个高级编 程模型, 程序员只需提供训练所需的初始输人、参 数和算法, 编程模型会自动实现算法加速模板的 配置.

(2) 设计生成器包括自动生成加速器及接口 逻辑.

(3) 预先设计的模板. 模板预先设计并且在所 有机器学习算法中是统一的. 支持所有在 TABLA 编程接口中定义的语言结构.

\section{TABLA 平台的局限性}

TABLA 平台如图 1 所示, 在使用规模、调度 策略和数据传输等方面存在着较大局限性, 限制 了其在深度学习算法加速的应用.

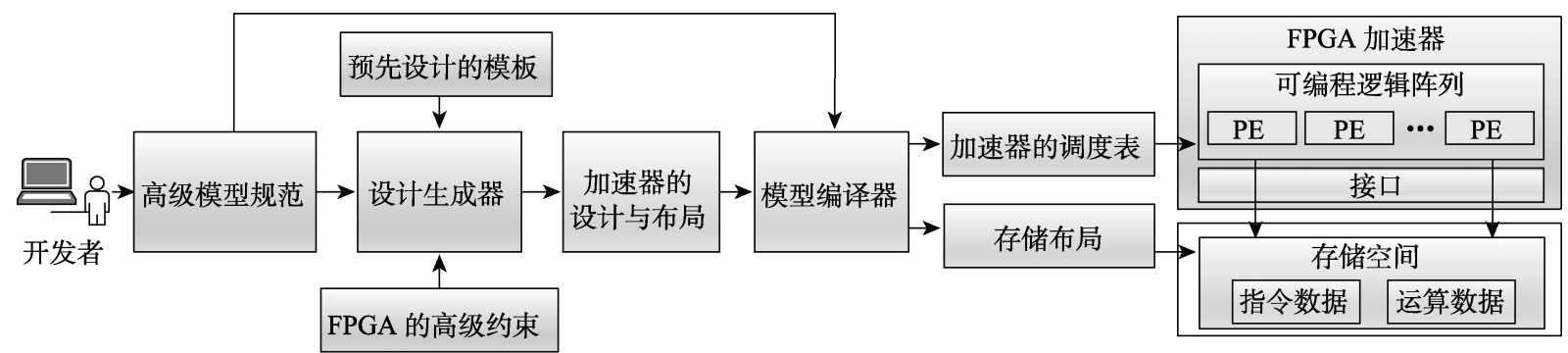

图 1 TABLA 平台

\section{1 调度策略}

调度加速单元(processing unit, PE)的策略要满 足以下约束条件和优化目标.

约束条件 1. 同一 PE 不能在一个周期内分配 给多个运算节点.
约束条件 2. 不能有运算节点未被分配 PE，分 配的 PE 不能超过资源总数.

优化目标: 在有多个 PE 满足约束条件时, 以 最小传输代价调度 PE.

约束条件 1 是硬件电路工作的基本条件，是时 
间和空间上的基本约束，一个 PE 在某一周期只能 处于一种状态或执行某一确定的运算功能，而不 能在某一周期处于多个状态或执行多个功能. 约 束条件 2 是硬件资源的限制，在有限的电路资源 下, PE 的个数与规模也会受到限制.

优化目标是最小传输代价. 最小传输代价是 指传输路径最短，运算的总体时间最少. 数据传 输的方式有 2 种, 一种为 PE 内部之间的数据传输, 这种传输方式不会发生阻塞, 而且传输的路径较 短; 另一种为 $\mathrm{PE}$ 之间的总线传输, 由于不同 $\mathrm{PE}$ 的总线优先级不同, 低优先级的 PE 在传输时会 出现等待、阻塞等情况, 因此这种方式传输的路 径较长.

TABLA 的调度策略较为简单, 并且调度策略 是针对某一特定的机器学习算法而设计的, 不具 备通用性. 采用的思路为先给最初始的运算节点 随机分配不同的 PE，再让剩余运算节点继承其父 节点的 PE，针对在特定算法中某些不能继承的节 点，再提出专门的优化策略. 以线性回归为例，该 机器学习算法的运算多为乘和累加, 所以每个运 算节点的输人个数为 2 , 输出个数为 1 , 如图 $2 \mathrm{a}$ 所 示. 这种数据流图复杂度很低. 由于运算节点输出 的个数为 1 , 用简单的基于继承的算法会自动满足 约束条件 1, 即让当前运算节点随机继承父节点的 PE，直到所有运算节点都被分配 PE.

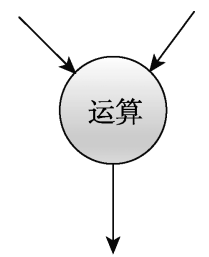

a. 单输出节点

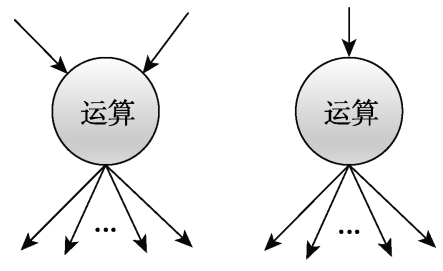

b. 多输出节点
图 2 运算节点

然而，该策略无法调度规模大且复杂的数据 流图, 如图神经网络 (graph convolutional networks, $\mathrm{GCN}$ ). 如图 $2 \mathrm{~b}$ 所示, GCN 中会出现大量的多输出 运算节点. 若沿用 TABLA 的调度算法会不满足约 束条件 2. 因为该节点的输出个数过多, 若其中 2 个 输出指向的运算节点在后续周期属于同一周期, 则 必然会使同一 PE 被分配多次, 产生硬件资源冲突.

\section{2 总线冲突与读冲突}

总线冲突. 对于大规模的运算, PE 的运算量 和数据传输量也是巨大的. 在大量数据传输时, 总 线的优先级会使某些低优先级的 PE 的运算结果不
能及时地传到其他 PE.

读冲突. TABLA 的设计是针对于传统的机器 学习算法. 机器学习算法的输人数据一般分为参 数型、常数型、数据型和梯度型等, 这些不同类型 的数据存储在硬件不同的随机存取存储器(random access memory, RAM)内. 在运算时 2 个输人的数 据来自不同的类型，故不会出现冲突. 但在复杂的 运算节点图(如 $\mathrm{GCN}$ )中会出现 2 个数据来自同一 类型，该情况下就会出现读冲突.

\section{3 总线优先级}

每个 PE 执行的是相应的指令文件, 该指令文 件是经过上层软件并行化分析后生成的数条指令. 理论上这些指令是有时间顺序的，但由于总线传 输有优先级, 数据传送到 PE 的顺序未必按照该 PE 指令执行的顺序, 这样在进行深度学习算法加速 的时候, 运算结果可能会出错. 以图 3 为例, 图中 的每个节点上的数字表示 PE 的标签, 该标签与 PE 一一对应. 标签越小, 表示总线传输优先级越高. 在该加速器运算时，在第 2 周期中标签为 1,2 和 3 的 PE 会传输数据给其他 PE. 标签为 1 的 PE 最先 传输, 标签为 3 的 $\mathrm{PE}$ 最后传输. 但标签为 3 的 $\mathrm{PE}$ (褐色节点), 需要先在第 3 周期处理第 2 周期标 签为 2 和 3 的 PE 传来的数据(图 3 中褐色线标出), 之后在第 5 周期处理第 2 周期标签为 1 的 PE 传来 的数据(图 3 中蓝色线标出). 由于总线优先级的原 因, 第 2 周期标签为 1 的 PE 传来的数据先到, 会 使标签为 3 的 PE 运算出错.

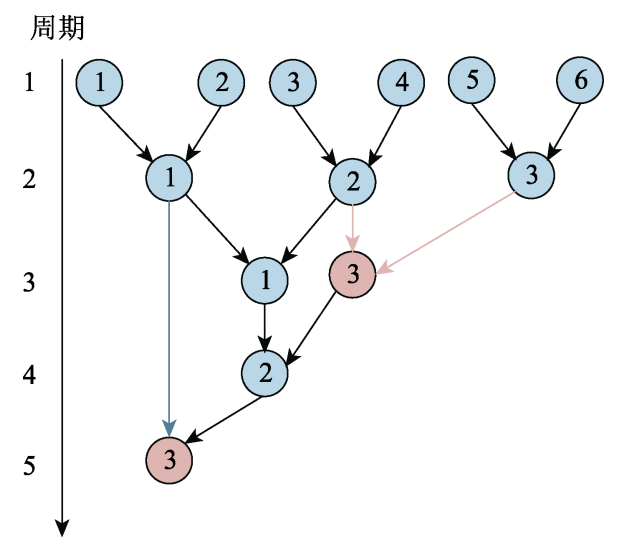

图 3 数据流图中依据总线优先级的数据传输

\section{4 应用规模}

TABLA 平台的核心 PE 类似于一个个小 CPU. 该单元的硬件功能存在冗余, 所以每个 PE 占用的 硬件资源较多. 在对每个数据流图配置 PE 时, PE 的规模必须与算法规模相适应. 若开发板没有足 
够的硬件资源去支持相应数量的 PE, TABLA 平台 是无法处理这种情况的, 这也是阻碍 TABLA 平台 推广到深度学习领域的主要原因. 为解决这些局 限性，本文在第 4 节提出了相应的解决方案.

\section{3 深度学习加速器框架设计}

\section{1 调度策略的优化}

为使 $\mathrm{PE}$ 支持深度学习算法，本文提出基于 $\mathrm{PE}$ 使用率和子节点与父节点之间继承关系的调度策 略. 具体算法如下所示.

算法. 基于使用率和节点继承关系的调度算法.

输人．运算节点集合 $N$ ，可调度的 PE $\boldsymbol{R}$

输出. 配置方案 $S$.

初始化. $i \leftarrow 1$.

Step1. 对初始运算节点进行加速单元的配置 $\boldsymbol{R}^{*} \leftarrow \boldsymbol{R}:$

Step1.1. 在初始运算节点选出运算节点 $n$, 在 $\boldsymbol{R}^{*}$ 随机选出一个 PE;

Step 1.2. 给该节点 $n$ 配置 PE, 将该 PE 使用率 加 1, 从集合 $\boldsymbol{R}^{*}$ 中删除该 PE;

Step1.3. 执行 Step1.1，直到所有初始节点被 配置.

Step2. 对距离初始节点距离为 $i$ 的运算节点调度 $\boldsymbol{R}^{*} \leftarrow \boldsymbol{R}:$

Step2.1. 选取距离初始节点为 $i$ 的运算节点 $n$; Step2.2. 若节点 $n$ 满足以下任意一个条件, 则 执行 Step2.3, 否则执行 Step2.4: (1) $n$ 的父节点有且仅 有一个子节点; (2) $n$ 的父节点有多个子节点且其他距 离初始节点为 $i$ 的节点未继承 $n$ 的父节点的 PE;

Step2.3. $n$ 节点继承其父节点的 PE;

Step2.4. 给 $n$ 节点配置使用率最低的 PE;

Step2.5. 被配置的 PE 使用率加 1, 从集合 $\boldsymbol{R}^{*}$ 中删除该 PE;

Step2.6. 执行 Step2.1, 直到所有距离初始节点 为 $i$ 的运算节点 $n$ 都被配置.

Step3. $i \leftarrow i+1$, 执行 Step2, 直到集合 $N$ 中的全 部运算节点被配置.

\section{2 总线冲突、读冲突的解决方案}

为了解决可能出现的总线冲突，增加了冲突控 制电路与寄存器单元, 将传输的 PE 的当前运行状 态保存, 直到总线没有被占用时再重新发送数据.

对于读冲突，将原先的执行周期再增加一周 期, 一共分为 3 个周期, 前 2 个周期读/写数据, 第 3 周期进行具体的运算, 并将这 3 步实现流水化.

\section{3 总线优先级的优化}

在规模较大并且复杂的运算中, 每个 PE 可能
会接收到在时域上无关的数据, 为了区别这些数 据还需要一些其他的信息. 所以本文把总线的位 数扩增 $n$ 位 ( $2^{n}$ 为 $\mathrm{PE}$ 总数). 这 $n$ 位信息用来表示 传输该数据的 PE 的信息, 作为标签对 PE 进行区 分, 如图 4 所示. 但在传输时也会有多个数据的标 签相同的情况，在运算时无法区分这些标签相同 的数据信息. 针对这种情况, 还需对同一标签的数 据进行时域上的区分. 本文根据上层编程模型的 工作特性设计出了相应的存储方案. 在最初 PE 调 度与指令文件生成的时候都是按照时间上的先后 关系, 依据这一特性, 将所有传输到达的数据依据 不同的标签存储在不同的 buffer 中. 在 PE工作时 从相应 buffer 中读取的数据即为此次运算所需要 的数据. buffer 先人先出的特性正好与数据先后使 用的特性相符合, 在这种存储方案下，不需要再设 计额外的控制电路, 只需依次从 buffer 中读取数据 即能保证运算结果的正确.

\begin{tabular}{|c|c|}
\multicolumn{1}{c|}{16 位 } & $n$ 位 \\
\hline 运算数据 & 扩展位 \\
\hline
\end{tabular}

图 4 扩展位数

假设每个 PE 需要增加的 buffer 个数为 $N(N$ 为 $\mathrm{PE}$ 的总数), 额外增加的 buffer 总数为 $N^{2}$, 如图 5 所示. 若 PE 的总数较多, 可能会消耗很多 buffer 资源. 此外, buffer 的深度决定了能容纳标签相同 的数据个数, 随着运算规模的扩大, 该 buffer 的深 度也要相应增加. 在本文中, 把 buffer 深度设置为 4 , 由此带来的存储开销为 $N^{2} \times(N+16)$.

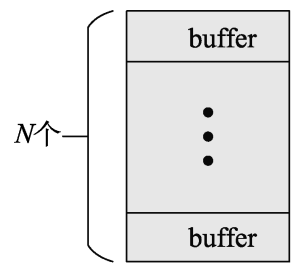

图 5 扩展 buffer

\section{4 硬件电路功能扩展与优化}

本文所提出的加速框架对 PE 的算术逻辑单元 (arithmetic and logic unit, ALU)模块增加了一些常 用的运算功能, 如深度学习中常用的 ReLU, Sigmoid 激活函数. 对于特定的深度学习算法, 如 $\mathrm{GCN}$, 需要求拉普拉斯矩阵, 本文增加了 $X^{-\frac{1}{2}}$ 和 $X^{-1}$ 的指数运算功能, 在设计这种较为复杂的运 算功能时采用查找表的方式. 
为使该加速平台能在有限的资源上工作，还 需提高资源的利用率. 假设结构和规模都是固定 的，一种有效地减少硬件资源使用的技术是量化. CPU 和 GPU 通常很少从量化中获益, 因为它们支 持一组固定的操作和数据类型, 而基于 FPGA 解决 方案通过量化技术可以实现更高的计算能效. 另 外, 对于 GCN 这种深度网络, 它的一个独特之处 在于, 它们往往能很好地应对输人中的高噪声. 这 种能力意味着它们可以把低精度的计算看做另一 种噪声的来源, 即使用包含较少信息的数字格式, 仍然可以产生准确的结果.

量化的方式可以采用开源的低精度矩阵乘法 库(general matrix to matrix multiplication low precision, GEMMLOWP). 通过内部使用 $b$ 位量化策略 避免运算的溢出. 为了将这些中间运算的结果重新 分配到 $0 \sim 2^{b}$ 的输出域, 本文采用了与 GEMMLOWP 相同的基于偏移/多重移位操作序列的方法. 若 $b=8$, 是指输人 8 位, 相乘后输出 8 位, 但中间运算 过程会按 32 位计算, 然后输出结果取 32 位的高 8 位; 否则, 中间运算若直接按 8 位算会溢出而损失 精度.

\section{GCN 加速}

深度学习算法的规模较机器学习相比要大得 多, 本文选择重量级深度学习算法 GCN 作为典型 例子, 说明本文的深度学习加速器框架设计所涉 及的关键技术.

\subsection{GCN 与 CORA 数据集}

近年来, 图上学习引起了人们的广泛关注. 通 过从非结构化高维图中提取结构化、低维特征, 可以 轻松地完成许多任务, 如节点分类 ${ }^{[21] 、}$ 链路预测 ${ }^{[22] 、}$ 图分类 ${ }^{[23]}$ 、聚类 ${ }^{[24]}$ 等. 在众多的具有代表性的学习 方法中, GCN 能够比传统方法更好地学习特征 ${ }^{[25-26]}$.

尽管 GCN 很受欢迎，但是在训练时间和计算 资源方面需要强大的算力. 目前如何利用 FPGA 硬 件加速训练的相关工作很少.

CORA 数据集是 $\mathrm{GCN}$ 的一种应用, 该数据集共 2708 个样本点, 每个样本点都是一篇科学论文, 所 有样本点被分为 8 个类别. 每篇论文都由一个 1433 维的词向量表示, 所以, 每个样本点具有 1433 个 特征向量, 特征向量为 0 或 1 , 共有 5429 个节点边.

$$
\boldsymbol{X}^{(i+1)}=\operatorname{ReLU}\left(\boldsymbol{L} \boldsymbol{X}^{(i)} \boldsymbol{W}^{(i)}\right)
$$

其中, $\boldsymbol{X}$ 为特征矩阵; $\boldsymbol{L}$ 为拉普拉斯矩阵,

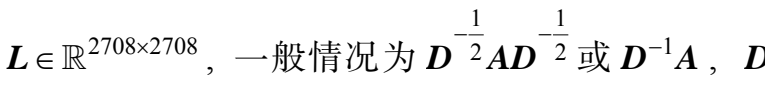
为节点图的度矩阵; $A$ 为节点图的邻接矩阵; $X \in$ $\mathbb{R}^{2708 \times 1433} ; W \in \mathbb{R}^{1433 \times f}$ 为权重矩阵; $f$ 为层数.

矩阵 $\boldsymbol{L X}$ 在经过数据流图的分析后, 有最多 $2708 \times 2708 \times 1433$ 个可以并行化的操作，即第 1 个 周期执行 $2708 \times 2708 \times 1433$ 次乘法，在之后的周期 对结果进行累加.

\section{2 可加速部分的提取}

以式(1)为例, 特征矩阵 $\boldsymbol{X}$ 与权重矩阵 $\boldsymbol{W}$ 的相 乘为主要耗时部分. 该部分是由大量的非 0 数进行 乘和累加运算, 运算量较大, 并且具有大量可以并 行化的操作, 即 $\mathrm{GCN}$ 传播公式中 $\boldsymbol{X}$ 矩阵与 $\boldsymbol{W}$ 矩 阵相乘适合于硬件上的加速.

GCN 的运算量很大，一般的 FPGA 开发板不 足以提供相应的硬件资源, 所以要对 $\mathrm{GCN}$ 算法先 进行算法层面的划分, 如将 GCN 训练切割成许多 小的运算, 加速器在进行每个小运算时提取该运 算的全部节点数据, 以这种方式来降低在开发板 资源上开销. 这种在算法层面分解的方式已在以 往的设计中取得了很好的效果 ${ }^{[27-28]}$.

GCN 在硬件加速上的重点应该在稠密矩阵的 乘法运算中. 针对稠密矩阵的乘法运算, 本文不采 取基于节点的关联性进行分解. 因为基于节点关 联性的分解主要应用于非欧几里得空间的不规则 图，在欧几里得空间的规则图上不具备可推广性. 本文采用一般的分块矩阵乘法运算.

分块矩阵乘法：设 $\boldsymbol{A}$ 为 $m \times l$ 阶矩阵, $\boldsymbol{B}$ 为 $l \times n$ 阶矩阵, 即 $\boldsymbol{A}$ 的列数等于 $\boldsymbol{B}$ 的行数, 分块 $\boldsymbol{A}=\left(\boldsymbol{A}_{[u, v]}\right)_{s \times r}, \boldsymbol{B}=\left(B_{[v, w]}\right)_{r \times t}$, 即 $\boldsymbol{A}$ 的列分块法等 于 $\boldsymbol{B}$ 的行分块法. 则 $\boldsymbol{A}$ 与 $\boldsymbol{B}$ 的乘积为 $s \times t$ 阶分块 矩阵

$$
\boldsymbol{C}_{[u, w]}=\sum_{v=1}^{r} \boldsymbol{A}_{[u, v]} \boldsymbol{B}_{[v, w]}
$$

其中, $u=1,2, \cdots, s ; w=1,2, \cdots, t$.

\subsection{GCN 模型编译器}

矩阵乘法分块进行模型编译器的处理后, 式(2) 的数据流图如图 6 所示. 该算法的最大并行度在第 2 周期, 横向并行化操作次数为 $r \times u \times v \times w$, 纵向周 期为 $M+m$, 横向并行化操作次数的 $r$ 参数会随着 原矩阵的改变而改变. 在 $\mathrm{GCN}$ 中该参数很大, 本文 期望将最大并行度稳定在一定范围内, 并且可以自 定义配置, 故对 TABLA 的数据流图的生成方式进 行修改. 将式(2)的数据流图的最大并行度通过矩阵 分解设置为 $u \times v \times w$, 周期设置为 $M \times r+m, u \times v \times w$ 


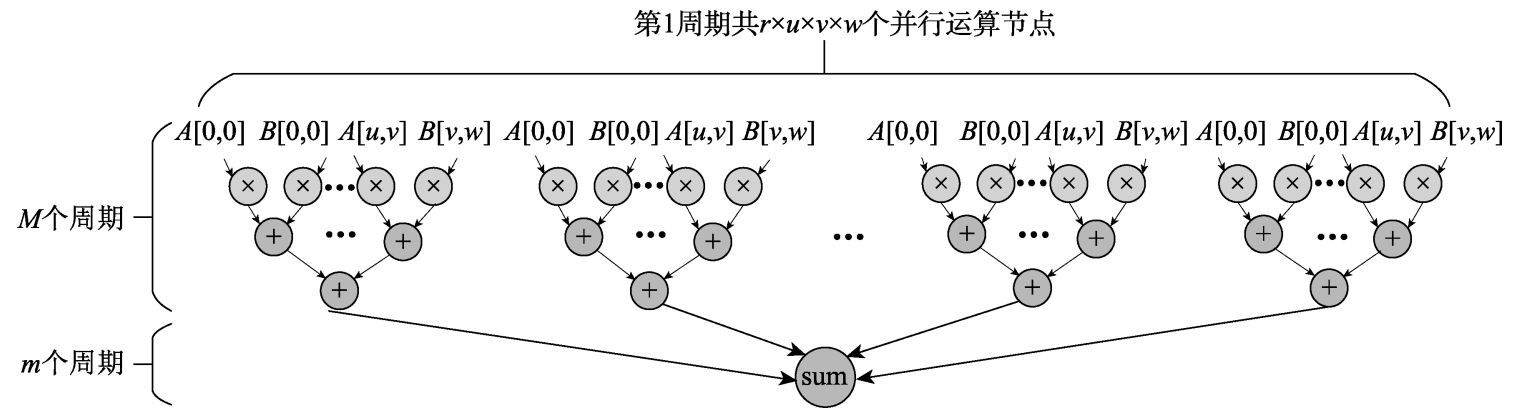

图 6 TABLA 平台输出的原始数据流

与原矩阵无关, 只与矩阵分块的大小有关. 生成数 据流图, 如图 7 所示. 该数据流图生成方式可以确 保横向的最大并行度, 并且该值不随原矩阵的改变 而改变，使设计更加具备通用性.

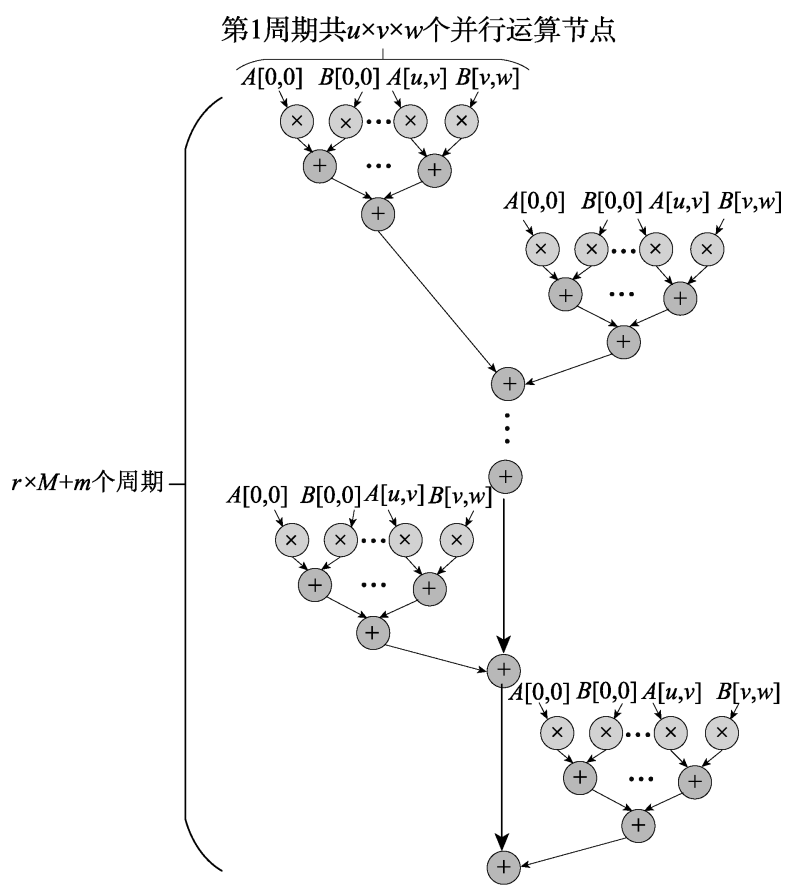

图 7 优化后的数据流图

本文在 ZYNQ-7020 开发板 ARM Cortex-A9 中 实现矩阵分块乘法的寻址运算、 $\mathrm{SD}$ 的数据与 FPGA
的通信. FPGA 进行 1 次矩阵运算的原始数据由该 CPU 通过 AXI(advanced extensible interface)总线传 输至 BRAM 中, FPGA 加速器直接从 BRAM 中读 取相应数据. 在读取的同时将 ROM 中的地址作为 导人的索引.

\section{4 片上与片外的数据交互}

本文采用的 ZYNQ-7020 系列开发板中, SD 卡具 备存储大量数据的能力, 故将 $\mathrm{GCN}$ 运算的全部数据 先存人 SD 卡中, 通过应用程序接口(application programming interface, API)将每次分块矩阵所需要的 数据读取到 FPGA 中. 该 API 的数据宽度为 32 位, FPGA 每次运算需要的数据与地址共为 67 位, 本 文将数据与地址分开存储, 每次矩阵运算的地址 顺序是相同的，因此将地址预先存储在只读存储 器(read-only memory, ROM)中.

\section{5 工作负载分配与协同}

本文采用 CPU-FPGA 异构架构, ZYNQ-7020 中有双核 ARM Cortex-A9 处理器 CPU. 图 8 为 $\mathrm{GCN}$ 加速平台的结构框图. 加速平台中的 ARM (advanced RISC machines)处理器实现高级模型编译器, 采用该高级模型编译器支持的自定义语法对算法 进行描述, 并对式(2)实现了数据流的分析, 从而 生成相应的数据流图, 提取并行化操作, 生成配置 加速器规模与 PE 命令的文件. 之后将相应的位流 文件通 Vivado 烧录至 ZYNQ-7020 开发板中.

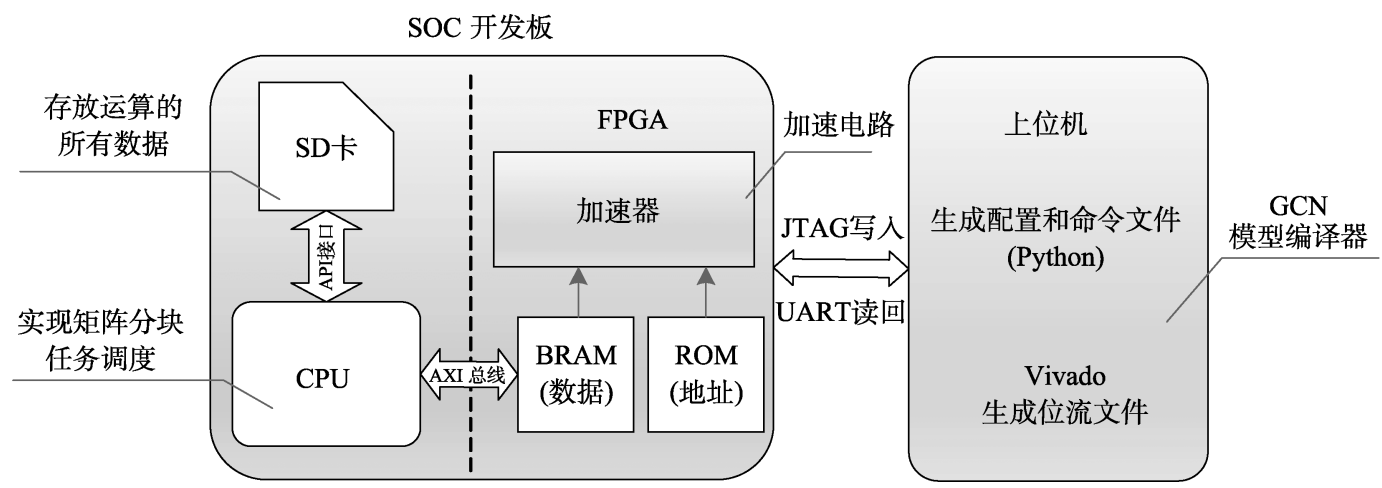

图 $8 \mathrm{GCN}$ 加速平台 


\section{5 实验及结果分析}

本文选用 Xilinx ZYNQ7000 开发平台完成板级

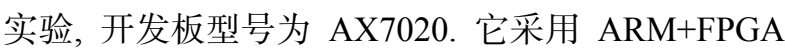
SOC 技术将双核 ARM Cortex-A9 和 FPGA 可编程逻 辑集成在一块芯片上. 它采用 Xilinx 的 ZYNQ7000 系列 XC7Z020-2CLG400I 作为核心处理器, 在 ARM 和 FPGA 上分别具有丰富的硬件资源和外围接口.

\section{1 加速器的资源占用量}

本文在 ZYNQ7020 开发板上测试了加速器的 资源使用情况, 每次测试都将分块矩阵的参数维度 进行修改, 表 1 所示为不同维度下测试最大并行度 的大小与资源的使用情况. 第 3 5 列为资源使用报 告，其中括号内为该 ZYNQ7020 开发板的最大资 源数.

表 1 加速器的 FPGA 资源使用情况

\begin{tabular}{cccccc}
\hline $\begin{array}{c}\text { 矩阵 } \\
\text { 维度 }\end{array}$ & $\begin{array}{c}\text { PE } \\
\text { 数量 }\end{array}$ & $\begin{array}{c}\text { Slice LUTs } \\
(53200)\end{array}$ & $\begin{array}{c}\text { Slice Registers } \\
(106400)\end{array}$ & $\begin{array}{c}\text { Block Bram } \\
\text { Tile(140) }\end{array}$ & $\begin{array}{c}\text { DSPs } \\
(220)\end{array}$ \\
\hline $2 \times 2$ & 8 & 4709 & 3614 & 10.0 & 8 \\
$3 \times 3$ & 27 & 11958 & 9005 & 31.5 & 27 \\
$4 \times 4$ & 64 & 21463 & 17652 & 86.2 & 64 \\
$5 \times 5$ & 125 & & & & \\
\hline
\end{tabular}

随着维度的增加, 加速器中 PE 的个数也随维 度的三次方形式增加. 对于 Slice LUTs 与 Slice Registers 资源，使用情况大致与 PE 的个数成正比. 该资源主要构成了 PE 中的各种逻辑控制电路, 很 难在综合中被优化. 对于 Block Bram Tile资源，该 资源主要构成了各 PE 中的存储资源, 第 3.3 节提 出的策略主要是由大量的 buffer 实现的, 每个 PE 中会插人 $N^{2}$ 个 $\operatorname{buffer}(N$ 为 $\mathrm{PE}$ 的总数), 随着 $\mathrm{PE}$ 的增加, buffer 的个数会随 2 次方的形式急剧增加, 这也是 Block Bram Tile 资源使用情况加速上升的 原因. 对于 DSP 资源，该资源完全与 PE 的个数成 一比一对应关系, 这是因为在分块矩阵乘法运算 中, 仅用到了乘和累加器, 其中一些不需要的运算 单元在综合时被优化了.

当分块矩阵的维度为 $5 \times 5$ 时, 最大并行度 $(\mathrm{PE}$ 的个数)达到了 125 , 该规模的加速器会在电路综 合时报错，该错误与资源的使用情况有关. 根据上 述分析，应该主要是由于 Block Bram Tile 资源不 够导致, PE 的增加导致 buffer 的个数急剧增加, 使 该开发板上的资源不足以支持如此多的存储需求. 综上所述, 即使 Slice LUTs 与 Slice Registers 的使 用情况没有超过总资源的一半，但是 Block Bram
Tile 资源已经超出该开发板的上限, 所以该加速器 的主要资源瓶颈为 Block Bram Tile.

\section{2 运行速度}

在开发板上进行实际的速度测试，测试的思 路为在保证运算结果正确的前提下，逐渐提高主 时频率, 直到出现时序约束不满足导致运算结果 出错的情况 (本文为 $1 \mathrm{GHz}$ ).

在保证运算结果正确的前提下，根据最高主 时钟频率 $f_{\text {max }}$ 与加速器的工作周期 $N_{\text {period }}$ 计算加 速的效果, $N_{\text {period }}$ 的值可以在仿真中得到. 具体计 算式为

$$
T=\frac{1}{f_{\max }} \times N_{\text {period }}
$$

将加速器在 FPGA 开发板中的运行速度与 CPU 的运行速度进行对比, 对比的计算机的型号为 OMEN HP laptop 15-dh0xxx, 处理器型号为 Intel $^{\circledR}$ Core $^{\mathrm{TM}}$ i7-9750 H CPU @ $2.60 \mathrm{GHz} 2.29 \mathrm{GHz}$, 内存 为 $16 \mathrm{~GB}$. 将所有的速度进行归一化处理, 让 CPU 的运行速度作为基数 1, 得到如图 9 所示的速度分 析. 图 9 中蓝色柱状图为经过板级验证得到的加速 效果, 灰色柱状图为 $5 \times 5$ 矩阵的加速提升, 该项未 进行板级验证，该值是假定主时钟频率为 $1 \mathrm{GHz}$ 时的仿真值.

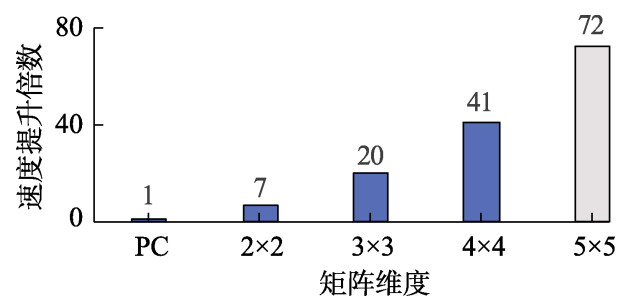

图 9 加速效果图

如图 9 所示, 随着矩阵维度的增加, 加速效果 显著. 在 $2 \times 2$ 的矩阵维度中有 8 个 $\mathrm{PE}$, 带来了 7 倍加速增益, 当矩阵维度提高到 $4 \times 4$ 时有 64 个 $\mathrm{PE}$, 带来了 41 倍的速度增益, PE 的个数与加速并行度 呈正相关. 矩阵维度增加使并行程度有很大的提 升. 理论上, 随着并行程度的增加, 加速效果应该 等比例增加, 但在图 9 中, 加速效果的增加速率随 着并行度的增加而降低. 这种情况是由于第 3.2 节 总线的等待机制造成的. 根据总线的协议, 任意周 期只能有一个 PE 占用总线并传输数据. 随着矩阵 维度越来越大, 数据的传输量也会显著增加, 这种 传输时的等待时间也会显著增加. 在较大维度的 矩阵运算中, 数据传输会成为该加速器的主要性 能瓶颈. 
图 10 所示为在配置不同 PE 个数下本文深度 学习加速器与 TABLA 平台对小规模矩阵运算的加 速效果对比, TABLA 平台由于对 PE 调度上的局限 性(见第 3.1 节), 生成的数据传输关键路径较长, 使其在调度 128 个 PE 时加速提升为负增长, 数据 传输成为主要的性能瓶颈. 总体而言, 本文设计的 深度学习加速器在加速性能上较 TABLA 平台有 $80 \%$ 的性能提升, 而且可以实现对深度学习加速 的支持.

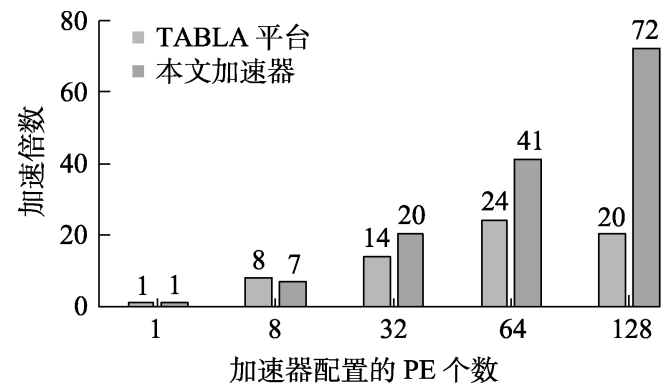

图 10 本文加速器与 TABLA 平台加速对比

\section{3 单位能效分析}

将本文的深度学习加速器的能效与 GPU 平台以 及 TABLA 相对比, 研究每瓦特功耗下较 CPU 相比性 能的提升，表 2 所示为实验中的 GPU 硬件信息.

表 2 实验中 GPU 型号及配置

\begin{tabular}{lccccc}
\hline \multicolumn{1}{c}{ 平台 } & 内核/个 & 时钟/MHz & 内存/GB & $\begin{array}{c}\text { 散热 } \\
\text { 功耗/W }\end{array}$ & 工艺 $/ \mathrm{nm}$ \\
\hline Tegra K1 & 192 & 852 & 2 & 5 & 28 \\
GTX650Ti & 768 & 928 & 1 & 110 & 28 \\
Tesla K40 & 2880 & 875 & 12 & 235 & 28 \\
\hline
\end{tabular}

图 11 所示为单位功耗下的加速性能提升, 本 文设计的深度学习加速器单位功耗的性能较 TABLA 平台有 $10 \%$ 左右的提升，与 GPU 相比有 3 4 倍的提升.

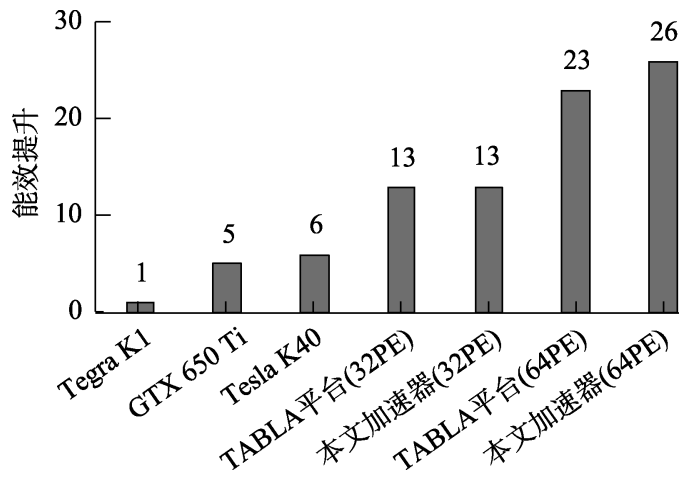

图 11 单位功耗下加速性能的提升

\section{6 结 语}

用 FPGA 加速深度学习算法是当下的热门方 向, 但这仍需要较长的开发周期和丰富的硬件设 计经验. 为了应对这一挑战, 本文设计了一种自适 应的深度学习算法加速平台. 本文首先分析了深 度学习算法硬件加速的可行性; 之后基于现有的 机器学习加速平台, 在应用规模、并行调度策略、 资源使用和功能扩展上进行了深人的研究, 并提 出了相应的优化策略. 本文采用 CPU-FPGA 异构 加速模板技术, 提出了自适应的上层模型编译框 架实现与不同硬件加速资源的适配. 这种基于定 制模板的软硬件协同设计可以很好地适配不同的 FPGA 芯片并支持算法的快速迭代. 在实验中，根 据不同 FPGA 的片上资源, 针对图神经网络学习算 法实现了与 CPU 相比 7 41 倍的速度提升.

\section{参考文献(References):}

[1] Smirnov E A, Timoshenko D M, Andrianov S N. Comparison of regularization methods for ImageNet classification with deep convolutional neural networks[J]. AASRI Procedia, 2014, 6(1): 89-94

[2] Qiu J T, Wang J, Yao S, et al. Going deeper with embedded FPGA platform for convolutional neural network[C] // Proceedings of the ACM/SIGDA International Symposium on Field-Programmable Gate Arrays. New York: ACM Press, 2016: 26-35

[3] Nomura O, Morie T. Projection-field-type VLSI convolutional neural networks using merged/mixed analog-digital approach[C] //Proceedings of International Conference on Neural Information Processing. Heidelberg: Springer, 2007: 1081-1090

[4] Chilimbi T, Suzue Y, Apacible J, et al. Project Adam: building an efficient and scalable deep learning training system[C] // Proceedings of the 11th USENIX Conference on Operating Systems Design and Implementation. New York: ACM Press, 2014: 571-582

[5] Zhang C, Li P, Sun G Y, et al. Optimizing FPGA-based accelerator design for deep convolutional neural networks[C] // Proceedings of the ACM/SIGDA International Symposium on Field-Programmable Gate Arrays. New York: ACM Press, 2015: $161-170$

[6] Esmaeilzadeh H, Sampson A, Ceze L, et al. Neural acceleration for general-purpose approximate programs $[\mathrm{J}]$. Communications of the ACM, 2015, 58(1): 105-115

[7] Han S, Liu X Y, Mao H Z, et al. EIE: efficient inference engine on compressed deep neural network[C] //Proceedings of the 43rd International Symposium on Computer Architecture. Los Alamitos: IEEE Computer Society Press, 2016: 243-254

[8] Du L, Du Y, Li Y L, et al. A reconfigurable streaming deep convolutional neural network accelerator for internet of things[J]. IEEE Transactions on Circuits and Systems I: Regu- 
lar Papers, 2018, 65(1): 198-208

[9] Misra J, Saha I. Artificial neural networks in hardware: a survey of two decades of progress[J]. Neurocomputing, 2010, 74(1-3): 239-255

[10] Vanderbauwhede W, Benkrid K. High-performance computing using FPGAs[M]. Heidelberg: Springer, 2013

[11] Putnam A, Caulfield A M, Chung E S, et al. A reconfigurable fabric for accelerating large-scale datacenter services[J]. Communications of the ACM, 2016, 59(11): 114-122

[12] Liang Y, Rupnow K, Li Y N, et al. High-level synthesis: productivity, performance, and software constraints[J]. Journal of Electrical and Computer Engineering, 2012, 2012(1): Article No.14

[13] Cong J, Liu B, Neuendorffer S, et al. High-level synthesis for FPGAs: from prototyping to deployment[J]. IEEE Transactions on Computer-Aided Design of Integrated Circuits and Systems, 2011, 30(4): 473-491

[14] Canis A, Choi J, Aldham M, et al. LegUp: high-level synthesis for FPGA-based processor/accelerator systems[C] // Proceedings of the 19th ACM/SIGDA International Symposium on Field Programmable Gate Arrays. New York: ACM Press, 2011: 33-36

[15] Mahajan D, Park J, Amaro E, et al. TABLA: a unified template-based framework for accelerating statistical machine learning[C] //Proceedings of the IEEE International Symposium on High Performance Computer Architecture. Los Alamitos: IEEE Computer Society Press, 2016: 14-26

[16] Zeng H Q, Prasanna V. GraphACT: accelerating GCN training on CPU-FPGA heterogeneous platforms[C] //Proceedings of the ACM/SIGDA International Symposium on Field-Programmable Gate Arrays. New York: ACM Press, 2020: 255-265

[17] Anguita D, Boni A, Ridella S. A digital architecture for support vector machines: theory, algorithm, and FPGA implementation[J]. IEEE Transactions on Neural Networks, 2003, 14(5): 993-1009

[18] Geng T, Wang T Q, Sanaullah A, et al. FPDeep: acceleration and load balancing of CNN training on FPGA clusters[C] // Proceedings of the 26th IEEE Annual International Symposium on Field-Programmable Custom Computing Machines. Los Alamitos: IEEE Computer Society Press, 2018: 81-84
[19] Guo K Y, Liang S, Yu J C, et al. Compressed CNN training with FPGA-based accelerator[C] //Proceedings of the ACM/ SIGDA International Symposium on Field-Programmable Gate Arrays. New York: ACM Press, 2019: 189

[20] Zhao W L, Fu H H, Luk W, et al. F-CNN: an FPGA-based framework for training convolutional neural networks[C] // Proceedings of the 27th IEEE International Conference on Application-specific Systems, Architectures and Processors. Los Alamitos: IEEE Computer Society Press, 2016: 107-114

[21] Hamilton W L, Ying R, Leskovec J. Inductive representation learning on large graphs[C] //Proceedings of the 31st International Conference on Neural Information Processing Systems. Cambridge: MIT Press, 2017: 1025-1035

[22] Zhang M H, Chen Y X. Link prediction based on graph neural networks[C] //Proceedings of the 32nd International Conference on Neural Information Processing Systems. Cambridge: MIT Press, 2018: 5171-5181

[23] Ying R, You J X, Morris C, et al. Hierarchical graph representation learning with differentiable pooling $[\mathrm{C}] / /$ Proceedings of the 32nd International Conference on Neural Information Processing Systems. Cambridge: MIT Press, 2018: 4805-4815

[24] Wang C, Pan S R, Long G D, et al. MGAE: marginalized graph autoencoder for graph clustering[C] //Proceedings of the ACM on Conference on Information and Knowledge Management. New York: ACM Press, 2017:889-898

[25] Grover A, Leskovec J. Node2Vec: scalable feature learning for networks[C] //Proceedings of the 22nd ACM SIGKDD International Conference on Knowledge Discovery and Data Mining. New York: ACM Press, 2016: 855-864

[26] Perozzi B, Al-Rfou R, Skiena S. DeepWalk: online learning of social representations[C] //Proceedings of the 20th ACM SIGKDD International Conference on Knowledge Discovery and Data Mining. New York: ACM Press, 2014: 701-710

[27] Huang W B, Zhang T, Rong Y, et al. Adaptive sampling towards fast graph representation learning[C] //Proceedings of the 32nd International Conference on Neural Information Processing Systems. Cambridge: MIT Press, 2018: 4563-4572

[28] Zeng H Q, Zhou H K, Srivastava A, et al. Accurate, efficient and scalable graph embedding[OL]. [2020-08-15]. https:// arxiv.org/pdf/1810.11899.pdf 